\title{
A network biology model of micronutrient related health
}

\author{
Ben van Ommen ${ }^{1}$, Susan Fairweather-Tait ${ }^{2}$, Andreas Freidig ${ }^{1}$, Alwine Kardinaal $^{1}$, Augustin Scalbert ${ }^{3}$ \\ and Suzan Wopereis ${ }^{1}$ \\ ${ }^{1}$ TNO Quality of Life, PO box 360, 4700 AJ Zeist, The Netherlands \\ ${ }^{2}$ University of East Anglia, Norwich NR4 7TJ, United Kingdom \\ ${ }^{3}$ INRA Centre de Recherche Clermont-Ferrand/Theix, 63122 St-Genès Champanelle, France
}

Micronutrients are involved in specific biochemical pathways and have dedicated functions in the body, but they are also interconnected in complex metabolic networks, such as oxidative-reductive and inflammatory pathways and hormonal regulation, in which the overarching function is to optimise health. Post-genomic technologies, in particular metabolomics and proteomics, both of which are appropriate for plasma samples, provide a new opportunity to study the metabolic effects of micronutrients in relation to optimal health. The study of micronutrient-related health status requires a combination of data on markers of dietary exposure, markers of target function and biological response, health status metabolites, and disease parameters. When these nutrient-centred and physiology/health-centred parameters are combined and studied using a systems biology approach with bioinformatics and multivariate statistical tools, it should be possible to generate a micronutrient phenotype database. From this we can explore external factors that define the phenotype, such as lifestage and lifestyle, and the impact of genotype, and the results can also be used to define micronutrient requirements and provide dietary advice. New mechanistic insights have already been developed using biological network models, for example genes and protein-protein interactions in the aetiology of type 2 diabetes mellitus. It is hoped that the challenge of applying this approach to micronutrients will, in time, result in a change from micronutrient oriented to a health oriented views and provide a more holistic understanding of the role played by multiple micronutrients in the maintenance of homeostasis and prevention of chronic disease, for example through their involvement in oxidation and inflammation.

Micronutrients: Homeostasis: Metabolomics: Phenotype: Health: nutritional systems biology

\section{Micronutrient supported metabolic health}

Many micronutrients are involved in the homeostatic regulation of overarching processes intimately involved in whole body metabolism, including oxidative-reductive and inflammatory pathways. Precise regulation of these processes is required to maintain the fine balance between optimal health and early onset of (diet-related) disease. Micronutrients act as cofactors in metabolic biochemistry (riboflavin, vitamin $\mathrm{B}_{12}$ ), and also in enzymes such as superoxide dismutase and glutathione peroxidase (GPX), that are involved in defense mechanisms ( $\mathrm{Se}, \mathrm{Zn}, \mathrm{Cu}, \mathrm{Fe}$ ). They have roles as chemical antioxidants (vitamins $\mathrm{C}, \mathrm{E}$ ), and some essential fatty acids are associated with protection against inflammation (EPA, DHA, ALA). Other micronutrients are involved in hormonal regulation, including iodine, which is essential for the biosynthesis of thyroxine $\left(\mathrm{T}_{4}\right)$ involved in metabolic regulation.

Thus, although each micronutrient is involved in one or more specific biochemical pathways and physiological functions and is ultimately associated with health problems with acute or chronic under- or overexposure; each interacts by modulating these or other pathways connected in a complex metabolic network essential for maintaining optimal health. The majority of micronutrient research to date has focused on the biological activity of single micronutrients or on the interactions between limited combinations of micronutrients. A more global view of their metabolic effects is therefore needed to appraise their impact on health.

With the development of systems biology (the study of multiple processes that together determine molecular/cellular and whole body physiology) we need to reconsider the function of micronutrients in the context of an integrated perspective. If all micronutrient-related processes are to work together and interact in order to maintain optimal health, it might be possible to examine their respective roles from the perspective of quantified metabolic health. A number of components of this quantification are described below, where the central theme is not to evaluate the individual components or parameters, but to focus on the systems approach. Four levels will be discussed, partly derived from the biomarker concepts established by Passclaim ${ }^{(1)}$ : (1) markers of exposure to food components; (2) markers of target function/biological response, which are usually functional parameters that are directly related to the micronutrient; (3) health parameters related to the micronutrient function, and (4) disease parameters related to micronutrient dysfunction. The Passclaim methodology deviates from the proposed structure, as it also uses intermediate biomarkers i.e. early observations related to disease onset, while we propose at this level to broaden the view from a micronutrient function perspective to a 
health perspective, exploiting new analytical and bioinformatics applications. We propose here to further extend the Passclaim concept by exploiting new analytical and bioinformatics methodologies in order to measure the effects of micronutrients on the global metabolic network. This should facilitate assessments of the impact on health at the individual level as well as taking into account interactions between micronutrients.

Currently, the assessment of dietary requirements is primarily based on the first and second parameters (micronutrient status), but, as yet, all of the information required for the complete process is not yet available. There are substantial gaps in our understanding of the metabolic role of many micronutrients, and current biomarkers are often inadequate to assess exposure and status. Nevertheless, the current enthusiasm for systems biology has stimulated a potential new approach for the quantification of dietary requirements based on all four types of parameters listed above. One of the recommendations made in the review of the UK Food Standards Agency Antioxidants in Food Programme ${ }^{(2)}$ was a broader view on mechanisms of action of functional effects and bioavailability of plant components (antioxidants) e.g. interactions between dietary factors and the immune system, markers of endothelial damage (e.g. ICAM-1, VCAM-1, P-selectin), modulation of Phase I and Phase II enzymes, and effects on gene expression and cell signaling. We now recognize that the relationship between micronutrients and health parameters ('overarching processes') is reciprocal, as changes in inflammatory status, for example, affect micronutrient bioavailability and efficacy ${ }^{(3)}$.

These arguments imply that all relevant parameters in micronutrient status, target function, and the impact on health and disease should be identified and integrated into a systems approach in order to evaluate relationships and ultimately to derive micronutrient recommendations for optimal health based on this new integrated approach. Analytical technologies are rapidly developing that can be used to quantify many of these parameters. Clearly, this approach is based on developments in nutritional systems biology, which aims to understand nutrient-health relationships at a systematic (molecular) physiology level, associating all relevant metabolic levels in a common network biology model ${ }^{(4-6)}$. With the possibility of quantifying gene transcripts (transcriptomics), proteins (proteomics), metabolites (metabolomics), as well as genotype variants (epigenetics) using post-genomic technologies, the technical goal should be within reach. In addition, basic systems biology research is providing tools and concepts in modeling and network biology. As this review focuses on the use of these technologies in network biology related to plasma measurements, emphasis is given to the metabolome and proteome rather than the transcriptome and epigenome which cannot be characterized in plasma. Addressing micronutrient requirements in relation to optimal health using these new approaches may seem like a quantum leap, but the steps required are rapidly becoming feasible, and this review proposes to provide an initial road map that will hopefully initiate discussion and stimulate nutritional scientists to devise experimental studies to test the hypothesis. In fact, similar visionary incentives have already been provided by senior nutrition scientists during recent years ${ }^{(7,8)}$. The advantages of this new approach are that firstly, it further embeds micronutrient biological activity in the context of molecular processes that are related to the maintenance of optimal health, and secondly, it enables us to describe and quantify the interactions between micronutrient in the same context. This review extends this avenue of thinking and proposes a series of applications related to this approach.

\section{Constructing the micronutrient biological network}

As mentioned above, the molecular components that together describe the relationship between micronutrients and metabolic health, as detected and quantified in plasma, can be grouped into four classes which are described below. Together, they comprise the components of the 'micronutrient biological network'.

\section{Micronutrient markers of exposure to food components}

Clearly, the concentration of individual micronutrients, their bioactive form, and specific biochemical/molecular markers or surrogate measures that reflect micronutrient intake and short-term status form the building blocks for more complex analyses involving metabolic pathways and multiple interactions. However, transient changes in plasma levels may have limited bearing on the longer-term impact on nutritional status, body pools and health. The supply of micronutrients that dictates plasma concentrations, enzyme-dependent levels and activities, and molecular markers is derived from two sources: dietary and endogenous. Mathematical models can be built to describe the flux of micronutrients between different body compartments, and the conduit for this process is the blood plasma, which is the reason why isotopes are employed to label the diet or endogenous pools in order to identify the origin of the micronutrient when attempting to study diet-health relationships. Another complication is that for a number of micronutrients, such as calcium, there is an overriding influence of homeostatic mechanisms that ensure that plasma concentrations are not perturbed but are maintained within a tightly controlled range.

\section{Markers of target function and biological response}

Functional biomarkers of micronutrients directly report on their primary biochemical role. Selenium is a cofactor in a number of enzymes, both with known and unknown function. Therefore, enzyme activities of glutathione peroxidase, thioredoxin reductase and 5-lipoxygenase $\mathrm{e}^{(9,10)}$ will be directly related to the quantity of available selenium in the body. Yet, other factors beyond selenium bioavailability influence the functional biomarker activity. For example, both genetic polymorphisms in 5-lipoxygenase ${ }^{(11-13)}$ and a series of GPX SNPs $^{(14,15)}$ have been reported. Furthermore, GPX enzyme induction as part of the oxidative stress response (exercise $)^{(16,17)}$ impacts on its activity and conversely selenium deficiency hampers GPX activity primarily under extreme oxidative stress conditions ${ }^{(18)}$. Interestingly, other micronutrients are also involved with the relationship between selenium and antioxidant capacity. Antioxidants such as ascorbic acid and alpha-tocopherol have a direct impact on antioxidant status and oxidative stress response. The enzyme cofactors $\mathrm{Cu}, \mathrm{Zn}$ and $\mathrm{Mn}$ influence superoxide dismutase activity, as do lipoic acid and ascorbic acid. Yet, Zn has many 
other targets that influence oxidative and inflammatory processes ${ }^{(19)}$. Parameters related to oxidative stress thus reflect the function of multiple micronutrients. Essential omega-3 fatty acids ALA, DHA, EPA have an impact on the integrity of the inflammatory machinery, and directly influence the selenium dependent 5-lipoxygenase activity. Thus, it is apparent that a biological network is beginning to unravel in which there are many interconnecting relationships whereby micronutrients have an impact on metabolic health. Furthermore, a number of phytochemicals have been reported to have an indirect antioxidant action, for example the isothiocyanate sulforaphane has been shown to interact synergistically with selenium $^{(20)}$. Synergy between sulforaphane and selenium in the induction of thioredoxin reductase 1 requires both transcriptional and translational modulation. Riboflavin, through its involvement in the biosynthesis of FMN and FAD, directly influences (energy) metabolism, as does niacin ${ }^{(21,22)}$. FAD is involved in folic acid metabolism as cofactor of MTFHR, thus linking to vitamin $\mathrm{B}_{12}$. In other words, many micronutrients are involved in the complex process of maintaining homeostasis through interconnected metabolic pathways.

\section{Micronutrient related health status metabolites}

The above discourse demonstrates that many micronutrients interact directly or indirectly in order to maintain homeostasis for overarching processes such as oxidation and inflammation, as well as being interdependent for many other metabolic pathways. Identification of the broader biological process that is reflected in the functional spectrum of any one micronutrient will demonstrate strong overlap with a number of other micronutrients as well as key phytochemicals. It thus makes sense to approach the functional assessment of micronutrient activity not only from a micronutrient perspective, but also from a health perspective, i.e. including all molecular/physiological processes that are influenced by the micronutrient. Quantification of the status of these overarching processes will complement the insights obtained from quantification of micronutrient status and functionality.

In extending this argument for selenium, we were able to detect in plasma not only the direct metabolite of the selenium dependent 5-lipoxygenase (5-HPETE), but also the four downstream leukotrienes (LTA4, LTC4, LTD4 and LTE4) (Fig. 1). These interact with a large family of oxylipids (prostaglandins, thromboxanes and lipoxins), of which current metabolomics technology can identify and quantify more than 80 different oxylipid members in one single LC-MS analysis ${ }^{(23)}$. In assessing these, we were able to quantify the impact of the omega-3 and omega- 6 fatty acids, together with the anti-inflammatory and anti-oxidant effects reported for alpha-tocopherol ${ }^{(24,25)}$.

Metabolomics technologies provide the opportunity to determine broad-brush assessments of a very large spectrum of plasma metabolites. GC-MS metabolomics now routinely encompasses the analysis of 250 identified and quantified plasma compounds, many of which directly reflect metabolic status $^{(26)}$. Lipidomics, the metabolomics branch dealing with lipophilic metabolites, also covers hundreds of compounds in plasma ${ }^{(27)}$. Databases give insight into the metabolome composition of plasma ${ }^{(28)}$. Thus, from a technological perspective, doors are opening to complement functional and targeted analysis with non-targeted 'open' analysis.
A major application of plasma metabolomics is differential display profiling, i.e. the comparison of the metabolomes of distinctly different phenotypic status (health/disease, lean/obese, low/high fat diet, etc). All metabolites are analysed without preconceived ideas of the differences in concentrations between phenotypes. Multivariate statistical analysis reveals a series of metabolites that are predictive for the differences, namely a 'biomarker profile'. Yet, metabolomics profiling is not straightforward and needs careful unraveling of biomarker profiles using multivariate statistical approaches. Mechanistic interpretation is needed as it will strengthen the statistical conclusions and reveal new insights. In the area of proteomics, this has lead to a series of diagnostic biomarker applications in the areas of cancer $^{(29,30)}$ and Alzheimer's disease ${ }^{(31)}$. Application of metabolomics in micronutrient related health status assessment thus allows an open and unbiased evaluation of metabolite changes that will complement the targeted approach described above. For example, comparison of plasma metabolomes in individuals exposed to high and low selenium intakes may reveal metabolite changes not previously described and this will add to the selenium plasma biological network and may also provide novel biomarkers of exposure. Of course the arguments presented above for metabolomics are equally valid for plasma proteomics and functional assays.

By using multiple comparisons, it will be possible to construct a series of connected micronutrient biological network models. Naturally, a note of caution is required because the 'omics' technologies mentioned are not yet mature in the sense that they do not provide yet a full coverage of identified and quantified metabolites and proteins. Yet, this molecular network approach is receiving broad attention in systems biology ${ }^{(31-34)}$, and nutrition research seems well positioned to profit from these developments.

\section{Micronutrient related disease parameters}

Micronutrient deficiencies have been associated with a series of pathologies such as hypertension ${ }^{(35)}$, colon and prostate cancer $^{(36)}$, breast cancer, CVD and osteoporosis. Yet, in many cases, our current knowledge does not allow us to make a causal link with the deficiency, although it may be described as a predisposing risk for the onset of the disease. It is largely accepted that a disturbance of metabolic homeostasis, oxidation and/or inflammation is a trigger for these diseases, and data linking modulation of these processes in (pre-) disease states to micronutrients have been published ${ }^{(24,25)}$. Yet, biomarkers or biomarker profiles related to these diseases might be partially relevant for (or contribute to) the micronutrient biological network, and thus, components may be added to the micronutrient biomarker profile. This group of biomarkers partly overlaps with the third group ('health related biomarkers'). Conceptually, a 'health space' can be defined as a multidimensional space built from concentrations of all metabolites or proteins constituting the metabolic network. The disease states may produce a biomarker profile in the extreme regions of the 'health space'. This 'health space' can be visualized by reducing it to two dimensions with statistical tools like principle component analysis, with all subjects positioned in this space based on the concentration values of all metabolites. 'Normal' subjects (with 'normal' values for all biomarkers) are projected in a defined zone, 

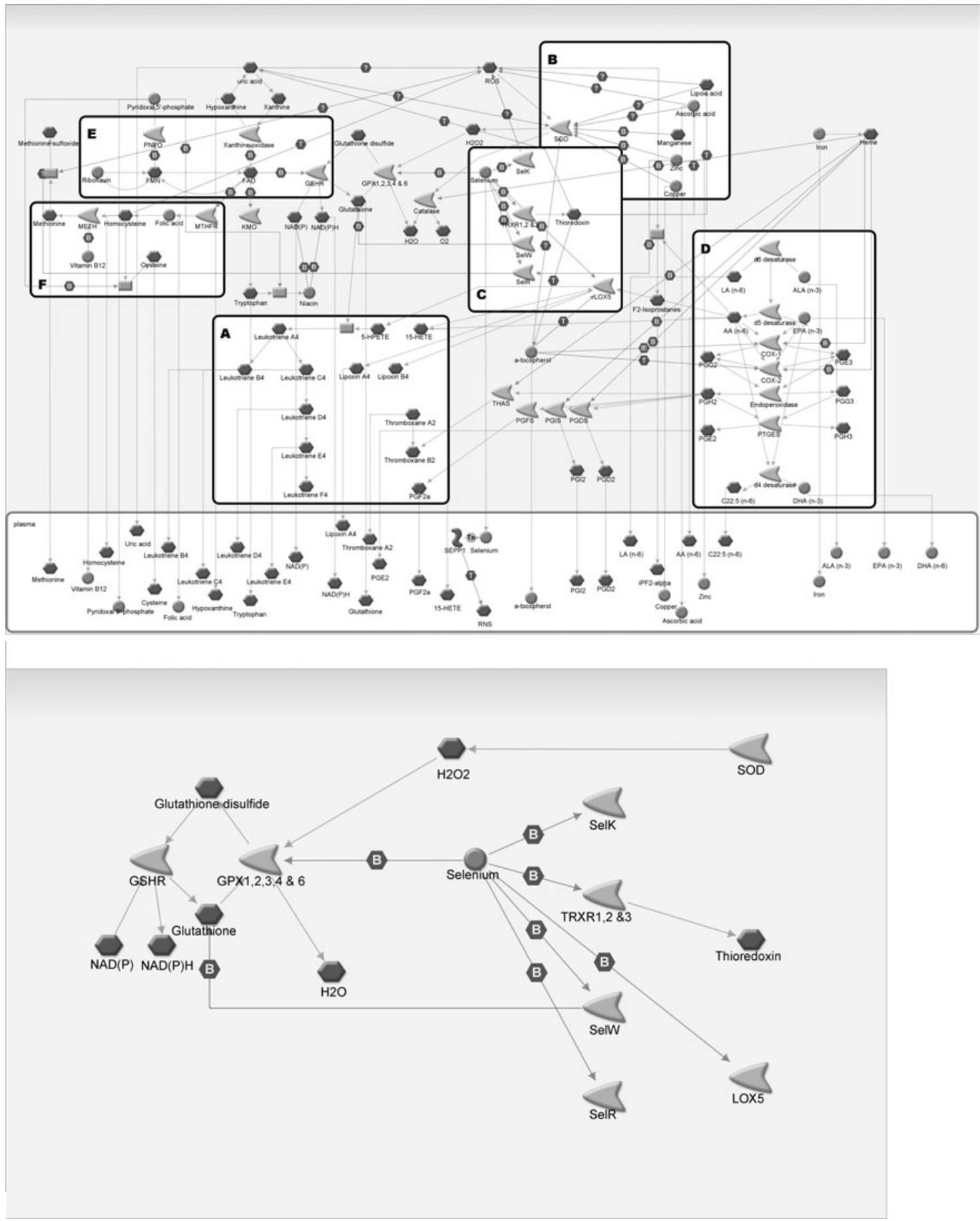

Fig. 1. The Selenium-centred micronutrient biological network. The most relevant biochemical processes related to selenium activity are presented in the context of metabolism, oxidation and inflammation, and embedded in the activity of micronutrients with similar activities. Also, the compartmental separation (intracellular vs. plasma) is presented, identifying the selenium centred plasma metabolome. The boxes identify various aspects of the selenium biological network: (A) the leukotriene processing as initiated by 5-lipoxygenase (LOX5), (B) the impact of various micronutrients on superoxide dismutase (SOD) which thereby infuences redox status and glutathione peroxidase (GPX) functioning, (C) the core selenoproteome, as presented separately in Figure 1, (D) the $n-3 / 6$ fatty acid machinery, $(\mathrm{E})$ the riboflavin, $\mathrm{FAD} / \mathrm{FMN}$ component, and $(\mathrm{F})$ the vitamin $\mathrm{B}_{12}$ and folic acid component. Symbols: hexagon, metabolite; circle, micronutrient; arrowhead, enzyme; red line, inhibition; green line, activation; B, binding; IE, influence on expression; T, transformation; ?, unspecified interaction. See http://www.eurreca.org/ everyone/4890

and aberrant subjects (diseased states) are projected in extreme regions out of the healthy zone. Similar concepts have been described for diseases and drug action ${ }^{(37,38)}$. Fig. 2 illustrates this concept.

\section{The micronutrient phenotype database}

The parameters of the micronutrient biological network, as derived from status, function and 'health' outlined above, together describe micronutrient-related health status. This 


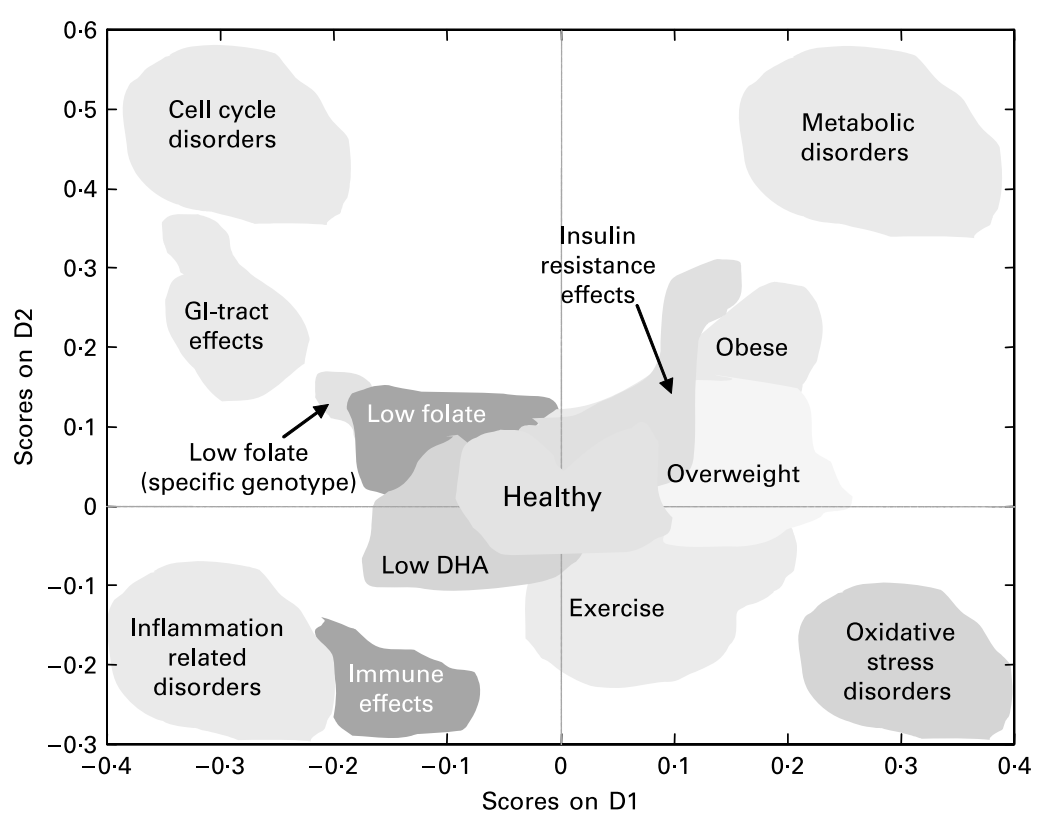

Fig. 2. Health space as defined by metabolic profiles. This (theroretial) two-dimensional plane is created by principal component analysis of all metabolite parameters affected by micronutrient status. A 'healthy phenotype' produces a metabolome profile concentrated around the center of the plan, while extremes are represented by disease states in which micronutrients play a role. Conceptually, these are arranged in metabolic effects, inflammation effects, oxidation effects and cell cycle effects. Nutrition related phenotypes (like obesity) show mild deviations from 'healthy', low levels of micronutrient supplementation (e.g. folate) demonstrates a biomarker shift from the healthy status, while on top of this a specific genotype may be visualized.

combines a nutrient centered view and a physiology or health centered view of the micronutrient and health relationship. Evaluation of these relationships provides a number of insights. Firstly, it allows the establishment of relationships between multiple micronutrient concentrations or status parameters and changing states of overarching processes. Secondly, it allows us to relate nutrient status, functional status and health status (individually and in combination) to a wide range of phenotypical, physiological and controlled dietary conditions. Thirdly, it may provide micronutrient recommendations based on the micronutrient biological network evaluation, i.e. a dietary recommendation based on a set of individual parameters.

A basic requirement for developing these insights is a streamlined method for capturing, annotating, storing, retrieving and interrogating these data. A database needs to be established that stores the status parameters described above together with accurate phenotypical and dietary quantification, the nutritional phenotype database. This concept has been introduced by previous authors ${ }^{(8,39)}$. The post-genomics scale expansion in biological sciences has triggered a large number of efforts to standardize, format and annotate parameters. The plasma metabolome has been disclosed by the Human Metabolome Project (HMDB, www.hmdb.ca), and a comparable community-based project is running for the human plasma proteome ${ }^{(40)}$. Ontologies are becoming available to describe physiological status and food/nutrient intake. The European Nutrigenomics Organization has developed tools to capture nutrition and health experimental annotations. A micronutrient nutritional phenotype database can thus easily be embedded (federated) in a number of ongoing projects. Furthermore, this strategy fulfils the requirement for increased transparency in reporting of human clinical studies ${ }^{(41)}$. The database needs to be a global consensus project allowing standardized collection and sharing of all relevant studies, and might actually be a driver for further experimental (analytical and procedural) standardization.

\section{Capturing requirements and variation in requirements with micronutrient biological networks}

External factors that impact on and hence determine the phenotype (e.g. life stage and life style, body weight etc, see Fig. 3) may affect micronutrient status and functionality, and thus affect the micronutrient-health relationship. Dietary requirements and recommendations for many phenotypes are being discussed and some have been established, based primarily on a variety of physiological parameters. Folate requirement in early pregnancy is increased due to the high rate of DNA synthesis in the embryo and this argument has led to specific recommendations. In addition, genetic variations have been described that are involved in folate metabolism and function ${ }^{(42)}$. Yet, these genetic differences cannot yet be translated into specific recommendations. A similar argument can be made for most micronutrients where there are well-established genetic disorders ${ }^{(43)}$, for example Wilson's disease, where copper accumulation results in hepatic toxicity. In addition, integrated approaches reveal unexpected links between copper metabolism, cell-cycle machinery, and cholesterol biosynthesis ${ }^{(44)}$. Consequently, it will be very difficult to dissect the human population into a number of subgroups with specific requirements due to the multitude of factors determining the phenotype, and the complexity of the related biology. An individual's health status (or phenotype) is the result of 


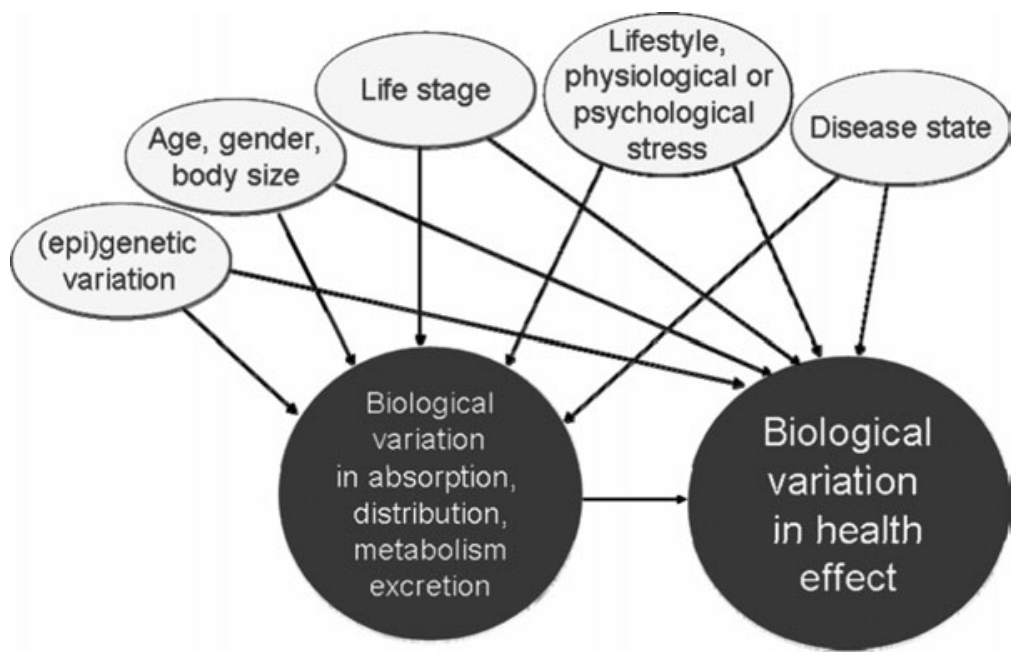

Fig. 3. Biological variations in health effects of micronutrients are partly related to intrinsic variation in bioavailability, and partly influenced by a range of other external factors (lifestyle, socio-economic status, etc). The assessment of variation in micronutrient needs can be made based on the quantification of all external factors. We propose to complement this approach by the quantifying health status, i.e. quantification of all relevant intrinsic phenotypical parameters instead of external environmental and exposure parameters.

many external (exposure, environment) and internal (genetics, ageing) factors. In the end, it will be very difficult to provide a dietary recommendation for all possible genetic variations and phenotypic states. Of course, pragmatic solutions exist, such as defining upper and lower limits together with the assessment of biological relevance in genetic variation. This approach, however, has a number of serious drawbacks. The folic acid story again illustrates this, with the numerous genetic polymorphisms involved and the practical impossibility to quantify the folate-health relationship for all relevant combinations of genotypic and phenotypic states ${ }^{(45,46)}$.

The primary argument in this review is that by assessing and correlating all relevant biological parameters (with an emphasis on plasma metabolites and proteins) that can be quantified, a holistic view on micronutrient function can be obtained and a series of parameters that drive variability (including genetics, epigenetics. and phenotypic origins of variation and vulnerability described above) may not necessarily need to be assessed, as the results of these drivers are quantified. Of course, a pragmatic approach would be to combine knowledge of drivers and results (e.g. a specific genotype with its effect on phenotype). Yet many examples are becoming available of very accurate 'omics-based' phenotypic characterizations, related to interventions ${ }^{(47-52)}$ and it is worthwhile pursuing this line of investigation, and developing and implementing the necessary toolbox.

Although we have made the above generalizations, we acknowledge that different phenotypes have different 'homeostatic values' for various metabolites in a micronutrient biological network. The 'one size fits all' approach will not work. And this is exactly the purpose of this 'micronutrient biological network profiling'. By using a large set of parameters, partly derived from scientific knowledge and partly from observations made in relation to various phenotypes, we can develop methodologies to correlate specific profiles (i.e. specific variations in multiple analytes) with specific phenotypes. A logical next step is the correlation of specific micronutrient(s) activities, with micronutrient requirements related to the phenotype.
A large body of research on variations in micronutrient status and functional parameters related to variation and requirements is available. An inventory could be made and captured in the nutritional phenotype database, and most likely, a series of variations in plasma parameters could be extracted which may be related to micronutrient-health relationships for specific genotypic and phenotypic conditions or subgroups. For each of the individual parameters, but more importantly, in a multivariate method, trends or profiles can then be described that are related to dietary requirements.

Next, in pursuing this approach, a broad inventory will be made of all relevant plasma parameters at biologically relevant lower and upper limit concentrations, and not just for those already known. Each component (metabolite) of the plasma network will be assigned a normal value and range related to a specific phenotype, derived from a series of sources (such as clinical chemistry databases, cohort studies, the Human Metabolome Database HMDB). In doing this, a careful match with phenotypic parameters needs to be made, and this requires a series of robust biomarkers that also describe early risk of chronic disease. This is a tremendous challenge because we not only want to describe a healthy phenotype for the purpose of quantifying the limits of 'health', but also to shed light on the risk of chronic disease and its prevention through diet. If, however, this worthwhile goal can be achieved, it would implicitly disclose the robustness of the micronutrient biological network, as the 'amplitudes' of the plasma parameters are correlated to vulnerability and unhealthy physiological states. Thus, insight will be obtained in the variations (upper/lower concentrations) in micronutrient plasma status and all related functional and 'health related' biomarker parameters that maintain a physiologically normal biological network, for any specific phenotype.

Application of biological network models has been shown to provide new mechanistic insights in relation to macronutrients. A network model of type 2 diabetes identified sets of genes and protein-protein interactions involved in the etiology of the disease. Moreover, different gene sets were observed to 
be related to different insulin resistance models ${ }^{(33)}$. Similar approaches (pathway network models) also have found their use in atherosclerosis research ${ }^{(53)}$; modern bioinformatics tools have been shown to be useful in constructing multidimensional biological relationships and have aided in understanding the complexity of health and disease relationships. The micronutrient biological network approach will thus profit from these applications in a holistic reassessment of the relationship between multiple micronutrients, metabolism, oxidation and inflammation. This relationship can easily be extended to bioactive phytochemicals (e.g. epigallocatechin gallate, resveratrol, lycopene) and bioactive lipids (e.g. omega-3/6 fatty acids).

\section{From micronutrient biological network to phenotype based micronutrient requirements: modeling and statistics}

The activities described above will provide network biology models which are 'parameterized' with normal values for a maximal number of metabolites and peptides. Also, where possible, these parameters will have information on how deviations in concentration impact (or at least are related to) deviations in health status. It is now possible to integrate statistical with biological evaluations. Multivariate statistical analysis of plasma metabolomes produce and exploit biomarker profiles, which can be matched with the plasma biological network. However, from a micronutrient point of view biomarker profiles need to be quantitative, comparable across studies and their components need to be interpretable within the biological context, if ever these profiles are to be accepted for providing guidance on interventions. This requires a careful selection of statistical methods, such as partial least square regression models (PLS) and well adapted normalisation approaches for data originating from different studies that feed the biological network. Bayesian network approaches have been applied in similar experiments (both genetics, transcriptomics and metabolomics and provide added value ${ }^{(54)}$. In fact, the 'health space' concept discussed above was developed for the pharmacological area using a Bayesian classifier approach ${ }^{(37)}$. Such approaches have been developed and validated in the field of genomics and metabolomics over the past 5 years and need to be adapted. This will create a combination of biological and statistical relationships, where certain parameters will be relatively stable and others will vary strongly based on specific external (nutrition, ...) or internal (health state, pre-disease state, ...) influences.

Next, a series of fine-tuning studies can be performed, that will serve to validate the quantitative model. Biomarker profiles related to micronutrient deficiencies or micronutrient related disease onsets can be obtained and matched with the network. Specific phenotypic or genotypic conditions which are expected to result in variations in micronutrient requirement can be entered into the model. The same can be done for studies where multiple micronutrients have been used. Such validation exercises increase the knowledge base linked to the micronutrient biological network.

Finally, the biological networks can be used in 'reverse mode', namely for predicting micronutrient requirements based on the profile of parameters of the micronutrient quantified by metabolome technology.

\section{How to begin?}

Achievement of such a major advancement in the field of micronutrient nutrition requires the building of an extensive knowledge base. As discussed previously, combined databases, common annotation and most of all a shared vision amongst scientists will be needed to drive this endeavour. From a number of fields in biology, it can be seen that the advantage of the technological leap of the last 10 years can only materialize if supported by strong consortia in the field (Human genome, yeast, C. elegans). Nevertheless, it should not be forgotten, that every journey starts with the first step and in this regard, this paper presents the vision for Micronutrient Network Biology within the EU Network of Excellence, EURRECA, a consortium of European Nutritional scientists ${ }^{(55)}$. Initial steps are being made within this consortium to commence data sharing that is necessary to build nutritional networks for micronutrients. Although many tools and solutions have become available within the public domain, there is still a gap for solutions dedicated to micronutrient biology and micronutrient studies. To develop this infrastructure, three stages will be implemented: (1) a combined database where scientists can contribute their study data in a controlled environment, (2) structured bioinformatics, specifically adapted for micronutrient biology, and (3) multivariate statistical tools, that build on the existing study design within the field, but can cope with the requirements of multiple linked response variables within the Biological Network.

\section{Acknowledgements}

All authors are member of the EU FP6 network of Excellence EURRECA (grant nr FP6-036196-2). This review was written as part of the work program of EURRECA. BvO, AF, AS and SW are member of the European Nutrigenomics Organisation (www.nugo.org). $\mathrm{BvO}$ and $\mathrm{SW}$ are members of the Netherlands Nutrigenomics Consortium. The authors thank Dr John Newman (USDA Davis, USA), Dr Chris Evelo (Un Maastricht, Netherlands) and Prof John Hesketh (University of Newcastle, UK) for their discussions on parts of the selenium biological network.

\section{References}

1. International Life Sciences Institute 2005, http://europe.ilsi.org/ activities/ecprojects/PASSCLAIM/.

2. Scientific Advisory Committee on Nutrition 2002, http://www. sacn.gov.uk/pdfs/sacn_02_18.pdf.

3. Pappa HM, Grand RJ \& Gordon CM (2006) Report on the vitamin $\mathrm{D}$ status of adult and pediatric patients with inflammatory bowel disease and its significance for bone health and disease. Inflamm Bowel Dis 12, 1162-1174.

4. Zhang X, Yap Y, Wei D, Chen G \& Chen F (2008) Novel omics technologies in nutrition research. Biotechnol Adv 26, 169-176.

5. Kussmann M, Raymond F \& Affolter M (2006) OMICS-driven biomarker discovery in nutrition and health. J Biotechnol 124, $758-787$.

6. van Ommen B (2004) Nutrigenomics: exploiting systems biology in the nutrition and health arenas. Nutrition 20, 4-8.

7. Young VR (2003) Trace element biology: the knowledge base and its application for the nutrition of individuals and populations. J Nutr 133, 1581S-1587S. 
8. Zeisel SH, Freake HC, Bauman DE, et al. (2005) The nutritional phenotype in the age of metabolomics. J Nutr 135, 1613-1616.

9. Dikiy A, Novoselov SV, Fomenko DE, et al. (2007) SelT, SelW, SelH, and Rdx12: genomics and molecular insights into the functions of selenoproteins of a novel thioredoxin-like family. Biochemistry 46, 6871-6882.

10. Papp LV, Lu J, Holmgren A \& Khanna KK (2007) From selenium to selenoproteins: synthesis, identity, and their role in human health. Antioxid Redox Signal 9, 775-806.

11. Bai C, Matsui E, Ohnishi H, Kimata K, Kasahara K, Kaneko H, Kato Z, Fukao T \& Kondo N (2008) A novel polymorphism, $\mathrm{E} 254 \mathrm{~K}$, in the 5-lipoxygenase gene associated with bronchial asthma. Int J Mol Med 21, 139-144.

12. Fowler SJ, Hall IP, Wilson AM, Wheatley AP \& Lipworth BJ (2002) 5-Lipoxygenase polymorphism and in-vivo response to leukotriene receptor antagonists. Eur J Clin Pharmacol 58, 187-190.

13. Kim SH, Bae JS, Suh CH, Nahm DH, Holloway JW \& Park HS (2005) Polymorphism of tandem repeat in promoter of 5-lipoxygenase in ASA-intolerant asthma: a positive association with airway hyperresponsiveness. Allergy 60, 760-765.

14. Foster CB, Aswath K, Chanock SJ, McKay HF \& Peters U (2006) Polymorphism analysis of six selenoprotein genes: support for a selective sweep at the glutathione peroxidase 1 locus (3p21) in Asian populations. BMC Genet 7, 56.

15. Villette S, Kyle JA, Brown KM, Pickard K, Milne JS, Nicol F, Arthur JR \& Hesketh JE (2002) A novel single nucleotide polymorphism in the $3^{\prime}$ untranslated region of human glutathione peroxidase 4 influences lipoxygenase metabolism. Blood Cells Mol Dis 29, 174-178.

16. Jozsi AC, Dupont-Versteegden EE, Taylor-Jones JM, Evans WJ, Trappe TA, Campbell WW \& Peterson CA (2000) Aged human muscle demonstrates an altered gene expression profile consistent with an impaired response to exercise. Mech Ageing Dev $\mathbf{1 2 0}$ $45-56$.

17. Ennezat PV, Malendowicz SL, Testa M, Colombo PC, Cohen-Solal A, Evans T \& LeJemtel TH (2001) Physical training in patients with chronic heart failure enhances the expression of genes encoding antioxidative enzymes. $J \mathrm{Am}$ Coll Cardiol 38, 194-198.

18. Palazzetti S, Rousseau AS, Richard MJ, Favier A \& Margaritis I (2004) Antioxidant supplementation preserves antioxidant response in physical training and low antioxidant intake. $\mathrm{Br} J$ Nutr 91, 91-100.

19. Zalewski PD (2006) Zinc metabolism in the airway: basic mechanisms and drug targets. Curr Opin Pharmacol 6, 237-243.

20. Zhang J, Svehlíková V, Bao Y, Howie AF, Beckett GJ \& Williamson G (2003) Synergy between sulforaphane and selenium in the induction of thioredoxin reductase 1 requires both transcriptional and translational modulation. Carcinogenesis 24, 497-503.

21. Kamanna VS \& Kashyap ML (2000) Mechanism of action of niacin on lipoprotein metabolism. Curr Atheroscler Rep 2, $36-46$.

22. Powers HJ (2003) Riboflavin (vitamin B-2) and health. Am J Clin Nutr 77, 1352-1360.

23. Newman JW, Kaysen GA, Hammock BD \& Shearer GC (2007) Proteinuria increases oxylipid concentrations in VLDL and HDL but not LDL particles in the rat. J Lipid Res 48, $1792-1800$.

24. Devaraj S, Tang R, dams-Huet B, Harris A, Seenivasan T, de Lemos JA \& Jialal I (2007) Effect of high-dose alpha-tocopherol supplementation on biomarkers of oxidative stress and inflammation and carotid atherosclerosis in patients with coronary artery disease. Am J Clin Nutr 86, 1392-1398.

25. Devaraj S, Leonard S, Traber MG \& Jialal I (2008) Gammatocopherol supplementation alone and in combination with alpha-tocopherol alters biomarkers of oxidative stress and inflammation in subjects with metabolic syndrome. Free Radic Biol Med 44, 1203-1208.

26. Kaddurah-Daouk R, Kristal BS \& Weinshilboum RM (2008) Metabolomics: a global biochemical approach to drug response and disease. Ann Rev Pharmacol Toxicol 48, 653-683.

27. Han X (2007) An update on lipidomics: progress and application in biomarker and drug development. Curr Opin Mol Ther 9, 586-591.

28. Wishart DS, Tzur D, Knox C, et al. (2007) HMDB: the Human Metabolome Database. Nucleic Acids Res 35, D521-D526.

29. Bonney GK, Craven RA, Prasad R, Melcher AF, Selby PJ \& Banks RE (2008) Circulating markers of biliary malignancy: opportunities in proteomics? Lancet Oncol 9, 149-158.

30. Liu XP, Shen J, Li ZF, Yan L \& Gu J (2006) A serum proteomic pattern for the detection of colorectal adenocarcinoma using surface enhanced laser desorption and ionization mass spectrometry. Cancer Invest 24, 747-753.

31. Ray S, Britschgi M, Herbert C, et al. (2007) Classification and prediction of clinical Alzheimer's diagnosis based on plasma signaling proteins. Nat Med 13, 1359-1362.

32. Han JDJ (2008) Understanding biological functions through molecular networks. Cell Res 18, 224-237.

33. Liu M, Liberzon A, Kong SW, Lai WR, Park PJ, Kohane IS \& Kasif S (2007) Network-based analysis of affected biological processes in type 2 diabetes models. PLoS Genet 3, e96.

34. Nikiforova VJ \& Willmitzer L (2007) Network visualization and network analysis. EXS 97, 245-275.

35. Chiplonkar SA, Agte VV, Tarwadi KV, Paknikar KM \& Diwate UP (2004) Micronutrient deficiencies as predisposing factors for hypertension in lacto-vegetarian Indian adults. $J$ Am Coll Nutr 23, 239-247.

36. Gross MD (2005) Vitamin D and calcium in the prevention of prostate and colon cancer: new approaches for the identification of needs. J Nutr 135, 326-331.

37. Paolini G, Shapland R, van Hoorn W, Mason J \& Hopkins A (2006) Global mapping of pharmacological space. Nat Biotechnol 24, 805-815.

38. Lamb J (2007) The connectivity map: a new tool for biomedical research. Nat Rev Cancer 7, 904-960.

39. German JB, Watkins SM \& Fay LB (2005) Metabolomics in practice: emerging knowledge to guide future dietetic advice toward individualized health. $J$ Am Diet Assoc $\mathbf{1 0 5}$ $1425-1432$

40. Omenn GS, Aebersold R \& Paik YK (2007) HUPO Plasma Proteome Project 2007 Workshop Report. Mol Cell Proteomics 6, 2252-2253.

41. Zarin DA \& Tse T (2008) Medicine. Moving toward transparency of clinical trials. Science 319, 1340-1342.

42. van der Linden IJM, Afman LA, Heil SG \& Blom HJ (2006) Genetic variation in genes of folate metabolism and neuraltube defect risk. Proc Nutr Soc 65, 204-215.

43. Montero R, Pineda M, Aracil A, Vilaseca MA, Briones P, Sánchez-Alcázar JA, Navas P \& Artuch R (2007) Clinical, biochemical and molecular aspects of cerebellar ataxia and Coenzyme Q10 deficiency. Cerebellum 6, 118-122.

44. Huster D \& Lutsenko S (2007) Wilson disease: not just a copper disorder. Analysis of a Wilson disease model demonstrates the link between copper and lipid metabolism. Mol Biosyst 3, $816-824$

45. Kohlmeier M, da Costa KA, Fischer LM \& Zeisel SH (2005) Genetic variation of folate-mediated one-carbon transfer pathway predicts susceptibility to choline deficiency in humans. Proc Natl Acad Sci USA 102, 16025-16030.

46. Stover PJ (2006) Influence of human genetic variation on nutritional requirements. Am J Clin Nutr 83, 436S-442S. 
47. Bouwens M, Afman LA \& Müller M (2007) Fasting induces changes in peripheral blood mononuclear cell gene expression profiles related to increases in fatty acid beta-oxidation: functional role of peroxisome proliferator activated receptor alpha in human peripheral blood mononuclear cells. Am J Clin Nutr 86, 1515-1523.

48. Chen C, Shah YM, Morimura K, Krausz KW, Miyazaki M, Richardson TA, Morgan ET, Ntambi JM, Idle JR \& Gonzalez FJ (2008) Metabolomics reveals that hepatic stearoyl-CoA desaturase 1 downregulation exacerbates inflammation and acute colitis. Cell Metab 7, 135-147.

49. Fuchs D, Vafeiadou K, Hall WL, Daniel H, Williams CM, Schroot JH \& Wenzel U (2007) Proteomic biomarkers of peripheral blood mononuclear cells obtained from postmenopausal women undergoing an intervention with soy isoflavones. Am J Clin Nutr 86, 1369-1375.

50. Kleemann R, Verschuren L, van Erk MJ, et al. (2007) Atherosclerosis and liver inflammation induced by increased dietary cholesterol intake: a combined transcriptomics and metabolomics analysis. Genome Biol 8, R200.

51. Walsh MC, Brennan L, Malthouse JP, Roche HM \& Gibney MJ (2006) Effect of acute dietary standardization on the urinary, plasma, and salivary metabolomic profiles of healthy humans. Am J Clin Nutr 84, 531-539.

52. Walsh MC, Brennan L, Pujos-Guillot E, Sébédio JL, Scalbert A, Fagan A, Higgins DG \& Gibney MJ (2007) Influence of acute phytochemical intake on human urinary metabolomic profiles. Am J Clin Nutr 86, 1687-1693.

53. Ghazalpour A, Doss S, Yang X, Aten J, Toomey EM, Van Nas A, Wang S, Drake TA \& Lusis AJ (2004) Thematic review series: the pathogenesis of atherosclerosis. Toward a biological network for atherosclerosis. J Lipid Res 45, 1793-1805.

54. Geier F, Timmer J \& Fleck C (2007) Reconstructing gene-regulatory networks from time series, knock-out data, and prior knowledge. BMC Systems Biology 1, 11.

55. www.eurreca.org. 